\title{
Actitud de los estudiantes y de los profesores del Departamento de Pediatría de la Facultad de Medicina de la Universidad Nacional Mayor de San Marcos hacia la ética médica
}

Attitude of students and teachers of the Universidad Nacional Mayor de San Marcos School of Medicine Department of Pediatrics to medical ethics

\author{
Manuel Hernán Izaguirre Sotomayor ${ }^{1, a}$, Luis Américo Reátegui Guzmán ${ }^{1, b}$, \\ Hallder Mori Ramírez ${ }^{1, a}$, Fabricio Junior Robles Rodríguez ${ }^{1, c}$, Walter Junior \\ Sánchez Vilcapuma ${ }^{1, c}$ \\ Facultad de Medicina San Fernando, Universidad Nacional Mayor de San Marcos, Lima, Perú. \\ ${ }^{a}$ Profesor Principal, Departamento de Pediatría, Profesor Asociado, Departamento de Pediatría; \\ ${ }^{\mathrm{b}}$ Profesor Principal, Departamento de Pediatría; ${ }^{\circ}$ Estudiante.
}

\begin{abstract}
Resumen
Introducción. La ética se ocupa de la moral; por tanto, es utilizada para calificar los actos de las personas ejercidos en condición de plena libertad como buenos o malos, es decir, Ios vincula con lo que debe o no debe realizar. Objetivo. Conocer la actitud de estudiantes y profesores de pregrado del Departamento de Pediatría de la UNMSM, hacia la ética médica. Diseño. Investigación no experimental, transversal, de tipo descriptivo y correlacional, de diseño cuantitativo. Lugar. Departamento de Pediatria, Facultad de Medicina San Fernando, Universidad Nacional Mayor de San Marcos, Lima, Perú. Participantes. Estudiantes y docentes del Departamento de Pediatria. Intervenciones. El instrumento fue una escala de actitudes tipo Likert construido por los autores. Principales medidas de resultados: Actitud hacia la ética médica. Resultados. Los docentes (82\%) tuvieron una actitud más favorable que los estudiantes $(48 \%)$ hacia la ética, sin embargo solo $36 \%$ de los docentes y $9 \%$ de los estudiantes tuvieron una actitud muy favorable. Conclusiones. Los docentes del Departamento de Pediatría tuvieron una actitud marcadamente más favorable que los estudiantes hacia la ética en general y una mayor satisfacción media hacia la deontología. Los docentes y estudiantes tuvieron una actitud desfavorable y muy desfavorable hacia el constructo la sociedad no es plenamente moral.
\end{abstract}

Palabras clave. Ética; Ética profesional.

\section{Abstract}

Introduction. Ethics deals with moral, and therefore is used to describe people's actions exercised in full freedom as good or bad, that is to say linked to what should or should not be done. Objective. To determine attitude to medical ethics of undergraduate students and teachers of San Marcos Department of Pediatrics. Design. Non-experimental, cross-sectional, correlational descriptive quantitative study. Setting. Department of Pediatrics, San Fernando School of Medicine, Universidad Nacional Mayor de San Marcos. Lima. Peru. Participants. Students and teachers of the Pediatrics Department. Interventions. The instrument applied was a Likert type attitude scale constructed by the authors. Main outcome measures. Attitude to medical ethics. Results. Teachers' attitude towards ethics was more favorable $(82 \%)$ than that of the students $(48 \%)$. However, only $36 \%$ of teachers and $9 \%$ of students had a very favorable attitude. Conclusions. Teachers of the Department of Pediatrics had a more favorable attitude to ethics than the students' and more satisfaction towards deontology than the students. Teachers and students had an unfavorable and very unfavorable attitude towards the construct "society is not completely moral", respectively.

Keywords. Ethics; Ethics, Professional.

An Fac med. 2015;76(4):361-8 / http://dx.doi.org/10.15381/anales.v76i4.11405

\section{INTRODUCCIÓN}

Se considera a la ética como la disciplina que se ocupa de la moral, y por lo tanto se utiliza para calificar a los actos de las personas, ejercidos en condición de su plena libertad, como buenos o malos, vinculado con lo que debe o no debe realizar. Es decir, la ética es la ciencia de la moralidad del accionar humano, contrariamente a la moral que al estar vinculada a las costumbres y al concepto de lo bueno y de lo malo no es única sino es cambiante, contrariamente a la ética que lo es. En este contexto, la ética médica se ocupa del estudio de los actos médicos profesionales frente al paciente y a la sociedad. Por lo tanto, debe tener cualidades específicas y requisitos, como conocer al detalle el código de ética ${ }^{(1)}$ de su profesión sobre el cual basará su actividad profesional, para lo cual el médico debe identificarse con los valores y principios morales que sustentan la ética médica. En este contexto, si los actos médicos realizados para cuidar o recuperar la salud son actos catalogados como buenos, entonces el acto médico cae en el ámbito de lo ético y lo moral. Como consecuencia, para el ejercicio médico es indispensable que a los estudiantes de medicina se les eduque dentro de los principios de la ética médica enseñándoles el código de ética que rige el ejercicio de la profesión médica en lo referente al ejercicio profesional y a la investigación científica ${ }^{(2)}$. 
En este sentido, según Perales y Mendoza (2009) ${ }^{(3)}$ uno de los factores que influye y muchas veces desencadena trasgresiones a la ética de los profesionales y estudiantes de la salud es la corrupción imperante en el país que se ha enraizado como expresión cultural de la población, corroyendo severamente los cimientos mismos de la institucionalidad legal del país, generando un clima emocional inadecuado en toda la sociedad, pervirtiendo las normas de convivencia individual y profesional preestablecidos, influyendo negativamente en el desarrollo y la productividad del país ${ }^{(4,5)}$.

Por lo tanto, los profesionales de la salud tienen que lidiar con el riesgo de infringir las normas éticas que rigen el ejercicio de la profesión médica, produciendo trasgresiones a corto, mediano y largo plazo para los profesionales involucrados, para la institución a la que pertenece y para la imagen percibida por la sociedad del médico, problema grave que ha despertado el interés mundial a lo largo de estas dos últimas décadas ${ }^{(6-7)}$.

Así mismo, al referirse a la corrupción y asistencia médica, Andrevski (1968) citado por Gonzales (1985) $)^{(3,8)}$ reveló que en algunos hospitales del África Occidental "Los médicos estatales cobraban por atender y hospitalizar a los pacientes (siendo la atención gratuita en tales centros); las enfermeras hacían lo mismo por alcanzarles las chatas; los empleados robaban medicamentos y los galenos, también; los pacientes indigentes recibían como único tratamiento inyecciones de agua coloreada y, a veces, también los que pagaban".

Precisamente, al otorgársele a los médicos prerrogativas muy específicas y muy bien delimitadas en el código de ética que constituyen el proceder adecuado del ejercicio profesional del médico sin olvidar el respeto a la dignidad del paciente, o realizando actos no médicos que generan daño al estado de salud al propiciar estados patológicos que pueden poner en peligro la vida de sus pacientes, no lo hace diferente a la ética en sí. Precisamente, los grandes progresos técnicos y clínicos de la medicina actual producen repercusiones morales sumamente complejas por sus implicancias morales, éticas, individuales y sociales. Por tal razón, los médicos tienen que tener una sólida formación moral para resolver los problemas éticos dentro del marco científico y ético, respetando la vida y la dignidad del ser humano ${ }^{(9)}$.

En este contexto, las Naciones Unidas, en 1982, estableció las normas para ser aplicadas por los médicos, al mismo tiempo que exhortaba a los gobiernos a que difundieran ampliamente los principios de la ética médica ${ }^{(10)}$.

Otro aspecto importante que la ética médica aborda es la relación médico-paciente para hacer conciencia en el médico que sus derechos frente al paciente se circunscribe a los que el paciente le da, implicando que arrogarse otros, por más buena intención del médico de mejorar algunos indicadores de salud, como ejercer control sobre el paciente 'medicalizando' su vida, o cuando no pone la docencia médica al servicio del enfermo no es ético ni mo$\mathrm{ral}^{(11-14)}$.

\section{MÉTODOS}

La investigación fue no experimental, transversal de tipo descriptivo y correlacional de diseño cuantitativo.

La muestra estuvo conformada por 96 estudiantes y por 46 docentes del Departamento de Pediatría, entre los meses de enero a diciembre del 2013.

Las unidades de análisis estuvieron conformadas por los estudiantes y por los docentes del Departamento de Pediatría.

$\mathrm{Al}$ no haberse utilizado métodos invasivos que pusieran en peligro la salud física y mental de los estudiantes y docentes, no existieron problemas éticos en el presente estudio. Sin embargo, se respetó los principios éticos aceptados por la sociedad, y los comités de ética de la universidad, así como la propiedad intelectual. La identidad de los estudiantes y de los profesores fue reemplazada por códigos al procesar y reportar los resultados.

El instrumento que se utilizó fue la escala de Likert confeccionada por los autores, el cual ha sido ampliamente empleado para evaluar la actitud de las personas en el Perú y el mundo. Fueron determinadas las actitudes tanto de estudiantes como de profesores frente a 21 constructos relacionados con la ética en salud.

La técnica utilizada fue la encuesta directa cara a cara. El instrumento usado para el recojo de los datos fue la escala de Likert que fue aplicada por un encuestador ajeno al servicio y capacitado en el manejo y procedimiento de su aplicación.

Los datos obtenidos fueron ingresados en Microsoft Office Excel 2000 y tabulados mediante software SPSS versión 15. Finalmente, se realizó el análisis descriptivo de todas las variables, a través de la determinación de proporciones de las variables sociodemográficas y de los 21 constructos mencionados.

En la ejecución de la investigación no se presentaron limitaciones importantes al desarrollo del mismo, salvo las inasistencias de los estudiantes al momento de responder la prueba y la negativa de los docentes a responderla. Lo que determinó la exclusión del estudio de varios estudiantes y docentes.

\section{RESULTADOS}

La actitud más favorable hacia la ética le correspondió a los docentes de sexo masculino, de 36 a 50 años, nombrados y con 1 a 20 años de experiencia, mientras que la actitud menos favorable hacia la ética le correspondió a los estudiantes de sexo femenino, de 36 a más años de edad y con 11 a más año de permanencia en la universidad. Así mismo, la actitud menos favorable le correspondió a las docentes mujeres 
mayores de 51 años, contratadas, y que tenían 21 a 30 años de experiencia, mientras que la actitud indiferente le correspondió a las estudiantes de sexo masculino, de 20 a 29 años de edad y con 1 a 10 años de permanencia en la universidad.

La actitud de los docentes hacia la ética médica fue favorable y muy favorable para $82 \%$, mientras que para los estudiantes fue $48 \%$. Contrariamente, $18 \%$ de los docentes tuvo una actitud indiferente, desfavorable o muy desfavorable hacia la ética médica, comparado con la actitud de los estudiantes que fue $52 \%$. (Figuras 1 y 2 ).

La actitud de los docentes hacia "Uno de los problemas morales en las instituciones más frecuente es la insuficiente dedicación al trabajo, ausencia del debido respeto a las personas y los conflictos frecuentes entre los miembros de la institución", "Los problemas morales en la atención a las personas más frecuentes son la ausencia de respeto a la dignidad, la autonomía y la integridad de las personas, deshumanización, despersonalización y comercialización de la atención a las personas" y "La ética es la teoría que explica todas las formas de conducta, personal o institucional, regulada por normas morales" fue favorable y muy favorable (86\%) para los tres ítems mencionados, mientras que la actitud desfavorable y muy desfavorable lo fue para el 11\% en el primero y $9 \%$ para el segundo. Mientras tanto para 9\% de los docentes la actitud fue muy desfavorable para el tercero.

Para estas mismas afirmaciones, la actitud de los estudiantes hacia el primer enunciado fue favorable y muy favorable para $94 \%$ de estudiantes y desfavorable y muy desfavorable para $2 \%$. La actitud hacia el segundo enunciado fue favorable y muy favorable para 93\% y desfavorable y muy desfavorable para $2 \%$. Mientras que la actitud hacia el tercer enunciado fue de favorable $y$ muy favorable $(86 \%)$, mientras que la actitud desfavorable y muy desfavorable lo fue para $11 \%$.

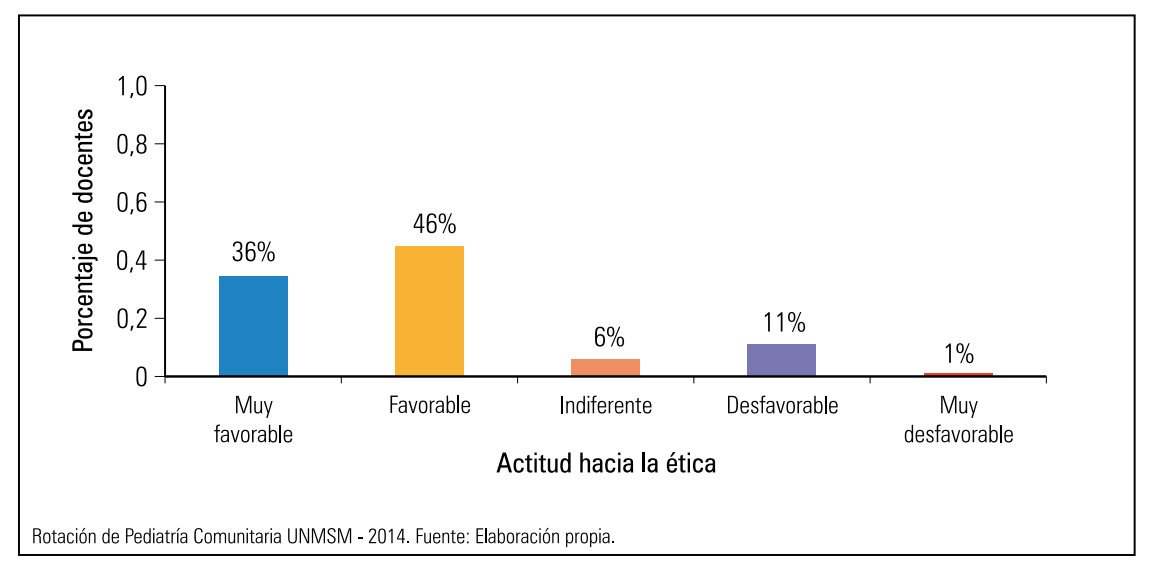

Figura 1. Actitud hacia la ética de los docentes de la rotación de Pediatría Comunitaria de la Facultad de Medicina.

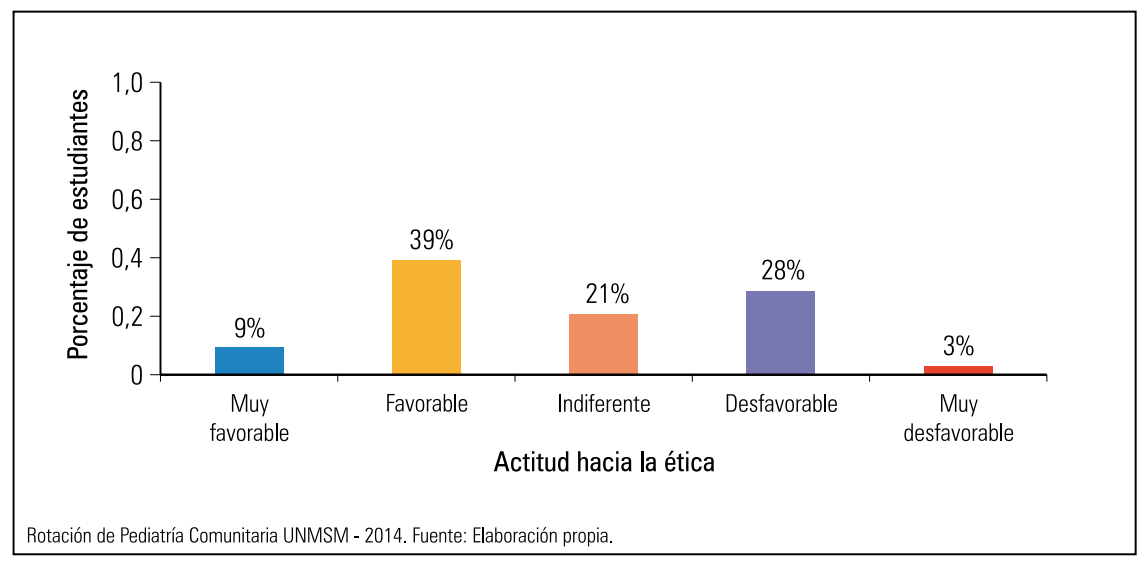

Figura 2. Actitud hacia la ética de los estudiantes de la rotación de Pediatría Comunitaria de la Facultad de Medicina.

La actitud para "La deontología es la teoría ética más aplicada al ejercicio profesional haciéndose explícita en los códigos de ética que contienen normas genéricas para todos los profesionales, y normas específicas según el tipo de trabajo de cada profesión" fue para el $47 \%$ de los docentes muy favorable y para el $53 \%$ fue favorable. La actitud de los estudiantes hacia este enunciado fue para $89 \%$ de los estudiantes muy favorable y favorable y para $3 \%$ desfavorable o muy desfavorable.

La actitud hacia "Los principios éticos se aplican como reglas para tomar decisiones" fue muy favorable y favorable para $79 \%$ de los docentes, y desfavorable y muy desfavorable para $19 \%$. Para el 93\% de los docentes, la actitud que tuvieron sobre los "Principios que deben orientar las relaciones entre personas son hacer el bien, no hacer daño, decir la verdad y mantener las promesas" fue muy favorable y favorable, mientras que para 5\% lo fue desfavorable y muy desfavorable. Sin embargo, la actitud hacia "La sociedad no es plenamente moral por que el hombre no es moral por naturaleza, porque la sociedad es estructuralmente inmoral y porque la teoría ética y las leyes no son suficientes" fue para 39\% de docentes muy favorable y favorable, mientras que para el $51 \%$ fue desfavorable y muy desfavorable.

Sin embargo, la actitud hacia el primer enunciado fue muy favorable y favorable para $73 \%$ de los estudiantes y desfavorable y muy desfavorable para 16\%. Para el $86 \%$ de los estudiantes la 
actitud que tuvieron sobre el segundo enunciado fue muy favorable y favorable, mientras que para el 3\% lo fue desfavorable y muy desfavorable. La actitud para el último enunciado fue para el $33 \%$ de estudiantes muy favorable y favorable, mientras que para $39 \%$ fue desfavorable y muy desfavorable y para $28 \%$, fue de indiferencia.

Por otro lado, la actitud del $77 \%$ de los docentes hacia "La conciencia moral se forma a edad temprana, y no cambia o cambia poco" fue muy favorable y favorable, siendo para el $21 \%$ desfavorable y muy desfavorable. La actitud hacia "Solo si la conciencia de una persona está moralizada, su comportamiento, su desempeño y su conducta será íntegramente moral" fue muy favorable y favorable para el $82 \%$, siendo muy desfavorable y desfavorable para 4\%. Mientras que la actitud hacia "La solidaridad es la adhesión a la causa o empresa de otros, la que determina finalmente la seguridad y la dignidad de las personas" fue muy favorable y favorable para $68 \%$ y desfavorable y muy desfavorable para 23\%. Finalmente, para el $81 \%$ de docentes su actitud fue muy favorable y favorable, y para $10 \%$ fue desfavorable y muy desfavorable para "La libertad es la facultad que tiene el hombre de obrar de una manera o de otra, y de no obrar, según sus capacidades por lo que es responsable de sus actos".

Para estos mismos constructos, la actitud de $48 \%$ de los estudiantes hacia el primer enunciado fue muy favorable y favorable, siendo para $31 \%$ desfavorable y muy desfavorable y para $21 \%$, de indiferencia. La actitud hacia el segundo enunciado fue muy favorable y favorable para el $68 \%$, siendo muy desfavorable y desfavorable para $16 \%$. Mientras que la actitud hacia el tercer enunciado fue muy favorable y favorable para $78 \%$, desfavorable y muy desfavorable para el $3 \%$ y para $19 \%$, de indiferencia. Finalmente, para el $86 \%$ de estudiantes su actitud fue muy favorable y favorable y para $7 \%$ fue desfavorable y muy desfavorable para el último enunciado. (Ver figuras 1 y 2).
La actitud de los docentes hacia "La justicia es la realización de las virtudes que inclinan a dar a cada quien lo que le corresponde o pertenece, en virtud a la realización de los deberes según los derechos de los otros" fue favorable y muy favorable para el $96 \%$, mientras fue desfavorable y muy desfavorable para $4 \%$. Para 93\% la actitud hacia "La ética científica debe ser el fundamento de todo el proceso educativo" fue favorable y muy favorable, siendo desfavorable y muy desfavorable para el 5\%. La actitud hacia "Dentro de la estructura tradicional, lo bueno es todo lo que uno haga por respetar la dignidad de los demás, hasta llegar a ser una persona digna para construir así una sociedad solidaria" fue favorable y muy favorable para el $90 \%$, siendo desfavorable y muy desfavorable para el 5\%.

Para estas mismas afirmaciones la actitud de los estudiantes hacia el primer enunciado fue favorable y muy favorable para $92 \%$, mientras fue desfavorable y muy desfavorable para 3\%. Para 83\%, la actitud hacia el segundo enunciado fue favorable y muy favorable, siendo desfavorable y muy desfavorable para el $3 \%$ y para el $14 \%$, de indiferencia. La actitud hacia el tercer enunciado fue favorable y muy favorable para el $90 \%$, siendo desfavorable y muy desfavorable para $1 \%$; para el $9 \%$ fue de indiferencia.

La actitud de los docentes hacia "Dentro de la estructura cultural, lo bueno es todo lo que uno haga por respetar la autonomía de los demás, hasta llegar a ser una persona autónoma para construir una sociedad libre" fue para $84 \%$ favorable y muy favorable y para $7 \%$ muy desfavorable. La actitud hacia "Dentro de la estructura económica, lo bueno es todo lo que uno haga por respetar la integridad de los demás, hasta llegar a ser una persona íntegra para construir una sociedad justa" fue favorable y muy desfavorable para $63 \%$ y desfavorable o muy desfavorable para $16 \%$; para $21 \%$ fue de indiferencia. Para $86 \%$ la actitud hacia "El estrés es una reacción particular entre el individuo y su entorno, evaluada por aquél como amenazante o desbordante de sus recursos poniendo en peligro su bienestar "fue favorable y muy favorable y para el $7 \%$ fue desfavorable o muy desfavorable.

Mientras que la actitud de los estudiantes hacia el primer enunciado fue para $75 \%$ favorable y muy favorable y para $4 \%$ desfavorable y muy desfavorable, para el $21 \%$ fue de indiferencia. La actitud hacia el segundo enunciado fue favorable y muy desfavorable para $70 \%$ y desfavorable o muy desfavorable para el $8 \%$ y para el $22 \%$, de indiferencia. Para $84 \%$ la actitud hacia el tercer enunciado fue favorable y muy favorable y para $6 \%$ fue desfavorable o muy desfavorable.

La actitud de los docentes hacia "La moral no consiste en lo que debemos hacer sino en por qué debemos hacerlo... Debemos crear una moral solidaria frente al mero acuerdo estratégico" fue para el $73 \%$ favorable y muy favorable y para 19\% muy desfavorable. La actitud hacia "La ética institucional es el conjunto de aspiraciones morales, normas, costumbres, creencias y otros, que dan forma a la cultura institucional de una institución" fue favorable y muy favorable para $97 \%$ y desfavorable y muy desfavorable para 3\%. La actitud hacia "Una de las estrategias de moralización social debería ser reforzar la ética institucional como mecanismo de defensa social, particularmente la solidaridad y vencer la desconfianza" fue favorable y muy favorable para $91 \%$ y para $9 \%$, de indiferencia. Finalmente, la actitud hacia "Se posee competencia moral cuando se tiene competencia moral y capacidad para actuar moralmente" fue favorable y muy favorable para $79 \%$ y desfavorable y muy desfavorable para $11 \%$.

Para los mismos constructos o afirmaciones, la actitud de los estudiantes hacia el primer enunciado fue para $76 \%$ favorable y muy favorable y para $10 \%$ desfavorable y muy desfavorable. La actitud hacia el segundo enunciado fue favorable y muy favorable para $90 \%$ y desfavorable y muy desfavorable para 
$6 \%$. La actitud hacia el tercer enunciado fue favorable y muy favorable para $88 \%$ y desfavorable y muy desfavorable para $4 \%$. Finalmente, la actitud hacia el último enunciado fue favorable $y$ muy favorable para $77 \%$ y desfavorable y muy desfavorable para 9\%; para el $15 \%$ fue de indiferencia.

\section{DISCUSIÓN}

La presente investigación pone de manifiesto que la actitud de los docentes del Departamento de Pediatría de la Facultad de Medicina de la Universidad Nacional Mayor de San Marcos hacia la ética fue para $82 \%$ favorable o muy favorable, lo que evidenciaría el interés y la predisposición de un accionar ético dentro de la institución educativa, contrariamente a la de los estudiantes que tuvieron una actitud favorable o muy favorable solo para el 48\%, existiendo más de la mitad que fueron indiferentes o tuvieron una actitud desfavorable o muy desfavorable, lo que explicaría lo que se constata a diario en las aulas cuando el estudiante es impuntual, copia durante los exámenes, miente en su afán de justificar sus deficiencias, entre otros.

Si bien es cierto, que docentes y estudiantes tienen una actitud favorable hacia la ética, esta es solo muy favorable para $9 \%$ de los estudiantes versus $36 \%$ de los docentes, a pesar que el mundo actual reclama una actitud muy favorable hacia la ética, toda vez que asistimos a una crisis de valores tanto a nivel local, regional y nacional, imposibilitando a que las instituciones educativas se conviertan en globalmente competitivas.

La actitud muy favorable y favorable de los docentes y de los estudiantes hacia los problemas morales en las instituciones más frecuentes, como la insuficiente dedicación al trabajo, ausencia del debido respeto a las personas y los conflictos frecuentes entre los miembros de la institución, así como a la ausencia de respeto a la dignidad, la autonomía, la integridad de las personas, deshumanización, despersonalización y comercialización del acto médico, se explicaría por la experiencia compartida al apoyarse entre ellos y a la relación de interdependencia que mantienen, convirtiéndolo en valor o principio para utilizarlo como mecanismo de defensa social, en su afán por mantener la cultura institucional sin cambios, siendo conscientes los docentes de su poco compromiso hacia la adecuada formación profesional y humana de los estudiantes y del poco esfuerzo y compromiso de los estudiantes hacia su propio aprendizaje.

En este sentido Ortiz ${ }^{(13,14,17,18)}$ remarcaba que "La ética social se transmite, como información social, a cada uno de los miembros de la sociedad a través del cerebro, transformándolo en individuo moral o persona”. Además precisaba que la poca dedicación al trabajo se explicitaba en la pobre dedicación a las obligaciones asignadas, la falta de cuidado en el cumplimiento de las tareas y a los elevados índices de impuntualidad". Precisamente este comportamiento moral observado constituye el "conjunto de aspiraciones morales, normas, costumbres, creencias y otros que dan forma a la cultura institucional" (3-5,16), evidenciando la importancia de cambiarlo si lo que se desea es convertir a la institución educativa en una institución moral y éticamente responsable.

La atención burocrática, deshumanizada, despersonalizada, aunado a la desidia, indolencia, negligencia ha derivado en la justificación de la comercialización de la atención médica hasta el punto de cosificarlo comparándola con un objeto dependiente de la oferta y la demanda del mercado sanitario.

La razón que el total de los docentes y el $89 \%$ de los estudiantes tengan una actitud muy favorable y favorable hacia la deontología y los códigos de ética que contienen las normas genéricas para todos los profesionales médicos traduciría el esfuerzo realizado por el Colegio Médico y la tendencia creciente en las instituciones de salud por recuperar el comportamiento moral de sus integrantes y de una explicación ética que lo sustente.

Así mismo, la actitud muy favorable y favorable de los docentes y estudiantes hacia los principios éticos para tomar decisiones, hacer el bien, no hacer daño, decir la verdad y mantener las promesas evidenciaría la importancia de los principios éticos como guía en el comportamiento de los profesionales médicos para beneficiar en toda decisión tomada al paciente, en el convencimiento que es la deontología la teoría ética más aplicada al ejercicio profesional que se hace explícita en los códigos de ética, a la vez que adopta carácter legal de cumplimiento obligatorio por todos los profesionales de la salud.

Sin embargo, la actitud desfavorable y muy desfavorable de los docentes y estudiantes hacia la sociedad no es plenamente moral porque el hombre no es moral por naturaleza, porque la sociedad es estructuralmente inmoral y porque la teoría ética y las leyes no son suficientes se explicaría en el pensamiento de Ortiz ${ }^{(13,14,17,18)}$ por la existencia de una incapacidad genética que impide comprender lo que debe ser, además que la "teoría del hombre no es correcta, la teoría ética no es lo suficientemente comprensiva, las normas morales no están definidas de modo adecuado, el sistema educativo es poco eficiente y hay sectores de la sociedad a los que no conviene una moral". Por consiguiente, enfatiza Ortiz que debe "reelaborarse la teoría del hombre, la teoría ética debe ser más comprensiva, las normas morales deben redefinirse, las tecnologías sociales deben reformularse y se debe luchar por la moralización social".

Por otro lado, la actitud muy favorable y favorable de los docentes y estudiantes hacia la conciencia moral se forma a edad temprana, y no cambia o cambia poco, y hacia solo si la conciencia de una persona está moralizada, su comportamiento, su desempeño y su conducta será íntegramente moral se sustentaría en que el hombre es un ani- 
mal, sabio, diestro, simbólico, religioso, económico y político; por tanto, su moral es individual dependiendo de sus genes o del ambiente, cuya conciencia moral se forma a edad temprana y no cambia o cambia poco ${ }^{(13,14,17,18)}$.

Mientras que la actitud muy favorable y favorable de docentes y estudiantes hacia la solidaridad es la adhesión a la causa o empresa de otros, la que determina finalmente la seguridad y la dignidad de las personas vendría a representar lo que Ortiz denomina el valor máximo de la solidaridad que se generó como valor máximo de la sociedad primitiva, además de contener un elemento afectivo de empatía o compasión que se genera en la interacción entre las personas.

Así mismo, el hecho que los docentes y estudiantes tuvieran una actitud muy favorable y favorable hacia la libertad es la facultad que tiene el hombre de obrar de una manera o de otra, y de no obrar, según sus capacidades por lo que es responsable de sus actos estaría relacionado a que la libertad determina la autonomía de la persona como expresión de la libertad social en cada individuo, dependiente de sus capacidades para decidir lo que es bueno para el trabajo, para los demás y para sí mismo, ser finalmente no esclavo, no estar sujeto o subordinado indebidamente, ni obligado a ciertos mandatos denigrantes ${ }^{(13,14,17,18) \text {. }}$

La actitud favorable y muy favorable de los docentes y estudiantes hacia la justicia es la realización de las virtudes que inclinan a dar a cada quien lo que le corresponde o pertenece, en virtud a que la realización de los deberes según los derechos de los otros y hacia la ética científica debe ser el fundamento de todo el proceso educativo y dentro de la estructura tradicional; lo bueno es todo lo que uno haga por respetar la dignidad de los demás, hasta llegar a ser una persona digna para construir así una sociedad solidaria estaría vinculada al convencimiento que la justicia determina la integridad de la persona, dado que según Ortiz la realización de los va- lores hace que una persona sea plenamente íntegra, corporal y moralmente, además la vigencia del derecho, la razón, la equidad, la realización de las virtudes que inclinan a dar a cada quien lo que le corresponde o pertenece y la realización de los deberes según los derechos de los otros es la que determina la integridad de la persona sustentada en la justicia que imparte la sociedad a la que pertenece.

Mientras que la actitud de los docentes y estudiantes hacia lo bueno es todo lo que uno haga por respetar la autonomía e integridad de los demás, hasta llegar a ser una persona autónoma e íntegra para construir una sociedad libre y justa estaría sustentada en que lo bueno es el eje de la realización de los valores, como lo sostenía Ortiz al enfatizar que "dentro de la estructura tradicional, lo bueno es todo lo que uno haga por respetar la dignidad de los demás, hasta llegar a ser una persona digna para construir así una sociedad solidaria, dentro de la estructura cultural lo bueno es todo lo que uno haga por respetar la autonomía de los demás, hasta llegar a ser una persona autónoma e íntegra para construir una sociedad libre y justa".

Por otro lado, la actitud favorable de los docentes y estudiantes hacia el estrés es una reacción particular entre el individuo y su entorno, evaluada por aquél como amenazante o desbordante de sus recursos poniendo en peligro su bienestar se debería, en la perspectiva de Perales ${ }^{(3-5,16)}$, porque cuando los "trabajadores perciben síntomas de ansiedad al acercarse al trabajo y de alivio al alejarse de él o cuando los trabajadores realizan sus funciones, en lo posible, individualmente, evitando meterse en problemas", reconocen que el aumento acelerado de "la comunicación anómala básicamente los chismes, así como los conflictos interpersonales" y el incremento de los problemas en el trabajo generan mayor estrés institucional.

Así mismo, la actitud muy favorable y favorable de los docentes y estudiantes hacia la moral no consiste en lo que debemos hacer sino en por qué debemos hacerlo; hacia la ética institucional es el conjunto de aspiraciones morales, normas, costumbres, creencias y otros, que dan forma a la cultura institucional; hacia una de las estrategias de moralización social debería ser reforzar la ética institucional como mecanismo de defensa social, particularmente la solidaridad y vencer la desconfianza y hacia se posee competencia moral cuando se tiene competencia moral y capacidad para actuar moralmente estaría en relación con lo que Perales ${ }^{(3.5,16)}$ definía lo que era la solidaridad institucional, la misma que era afectada por el estrés y con lo que Ortiz ${ }^{(13,14,17,18)}$ remarcaba que los principios morales sustentados por las teorías éticas en la historia de la humanidad no son sino la clase de información social que refleja las aspiraciones morales máximas de todos los pueblos: básicamente las aspiraciones de solidaridad, libertad y justicia y que la formación y la actuación moral de todas las personas es, entonces, responsabilidad de cada una de tales estructuras sociales donde vive y se forma cada personalidad".

El hecho que los estudiantes tengan una actitud más favorable que los docentes hacia la poca dedicación al trabajo, ausencia del debido respeto a las personas y los conflictos frecuentes como los problemas morales más frecuentes en las instituciones se explicaría por la poca motivación de los docentes a cumplir con sus actividades educativas presionados por sus obligaciones económicas que no pueden cubrirlos con sus honorarios en las instituciones educativas, obligándolos a buscar trabajos adicionales que le demandan tiempo y esfuerzo que los toma lamentablemente del tiempo que debería dedicarlo a su tarea educativa. Aunado a que el estrés que le produce esta situación se comporta conflictivamente y por lo tanto a veces no respeta a los demás. En este sentido Perales (3-5,16) identificó un ambiente de tensión que se objetiva particularmente en las reuniones de grupo, al igual la presencia de un "sentimiento de frustración y si- 
lencio resentido, manifiestos durante el desempeño de las tareas diarias aunado a un aumento de la comunicación anómala por medio del rumor y del chisme".

Situaciones como la descrita afectan sistemáticamente a los miembros de la institución en todos los niveles. El personal, sea individual o grupalmente, tendrá que hacerle frente con su propia capacidad de afronte. Dos opciones son claras: o el miembro se retira de un ambiente tan enrarecido o se adapta a él. Lo primero, no siempre es posible por la necesidad real de conservar el ingreso regular del puesto laboral. Ello aumenta la necesidad de adaptación, lo que implica aceptar, a nivel individual, las inconductas que se dan en la institución ${ }^{(3-5,16)}$.

Igualmente, la actitud más favorable que mostraron los estudiantes comparado con la actitud de los docentes hacia la falta de respeto a la dignidad, a la autonomía, a la integridad, a la deshumanización, a la despersonalización y a la comercialización de la atención a las personas se explicaría por la cosificación de la medicina al considerarla como un bien cualquiera sujeto a las leyes de la oferta y la demanda que ha llevado a que los estudiantes la consideren como algo natural, creencia cimentada por lo que presencian en sus prácticas respecto a la conducta de los docentes frente a la atención de los pacientes, a pesar que ambos tenían la misma actitud frente al concepto de la ética y la deontología. Situación que se ve agravada por el enfoque predominantemente técnico que se le da en la actualidad a la enseñanza médica, ignorando casi totalmente lo relacionado al espíritu del hombre, sabiendo que la ética es esencialmente espíritu.

Esta situación de tolerancia hacia la cosificación del acto médico y de la educación médica explica que solo un poco más del tercio de los estudiantes y la mitad de los docentes tengan una actitud desfavorable a considerar a la sociedad como no plenamente moral porque el hombre no lo es por natura- leza, dado que la sociedad es estructuralmente inmoral y porque la ética y las leyes no son suficientes al considerarla como una conducta cotidiana y normal que no es cuestionada por la gran mayoría de docentes y estudiantes. Percepción que se podrá revertir, como lo propone Perales ${ }^{(3)}$ si se logra "reforzar la reflexión ética y la formación moral en el proceso de educación médica, entendida no meramente como un proceso de nivel cognoscitivo o psicomotor, sino como un conjunto de experiencias y vivencias que se traduzcan en unas disposiciones afectivas y unas actitudes y formas de conducta que expresen el sentido de encuentro interpersonal que es inherente al acto médico" y a la relación docente discente.

Por otro lado, es comprensible que los docentes tengan una mejor actitud que los estudiantes frente a que solo si las personas son morales su comportamiento y desempeño también lo será dado que el docente es una personalidad ya formada moralmente, mientras que los estudiantes "dentro del sistema educativo, y durante su infancia, niñez y adolescencia, el individuo humano se transforma en una personalidad", así como el desarrollo ampliado de la personalidad madura, depende de su capacidad de autoformarse como trabajador social, a pesar de las condiciones negativas de la sociedad" $(13,14,17,18)$. Es más, Ortiz ${ }^{(13,14)}$ enfatizaba que la personalidad es cada individuo humano organizado a partir de la información genética de sus células y a base de la información psíquica consciente que refleja la sociedad donde se forma, produce, crea y se reproduce. Lo que se necesita para elegir un comportamiento correcto solo requiere discernir clara y coherentemente, pero lamentablemente no todos los docentes y estudiantes tienen una suficiente capacidad reflexiva como para formular correctamente juicios éticos.

Llama la atención que los estudiantes tengan una actitud más favorable que los docentes hacia el concepto de solidaridad, a pesar que los docentes por su propia formación profesional y social deberían no solo tener el concepto de solidaridad, sino de serlo dado que para Ortiz la solidaridad es un valor social y la dignidad es un valor personal, que se erigió como el valor máximo de la sociedad primitiva conteniendo un elemento afectivo de empatía o compasión generada en la interacción entre las personas para determinar la seguridad y la dignidad de las personas, adaptándose el comportamiento de los mismos a la estructura tradicional imperante.

Contrariamente a lo que transmiten aparentemente los docentes con su comportamiento en las instituciones educativas, tuvieron una actitud más favorable que los estudiantes hacia que la ética científica debe ser el fundamento de todo el proceso educativo, toda vez que según Ortiz ${ }^{(13,14,17,18)}$ la conducta del docente y los estudiantes se evidencia en problemas del rendimiento, del uso del tiempo y de los contenidos, de las disposiciones frente al paciente y su familia, de las aptitudes para la solución de problemas del paciente, entre otros que están vinculados más con las actividades cotidianas de los docentes que al de los estudiantes, a pesar que la actitud de ambos frente a que lo bueno es todo lo que uno haga por respetar la dignidad de los demás, hasta llegar ser una persona digna para construir así una sociedad solidaria.

La explicación del por qué los docentes $(82 \%)$ tuvieron una actitud marcadamente más favorable que los estudiantes (48\%) hacia la ética en general y una mayor satisfacción media de los docentes hacia la deontología que los estudiantes se debería a que los docentes ya son personalidades ética y moralmente constituidas, además de ser conscientes de la deontología de la profesión médica que es la teoría ética más aplicada al ejercicio profesional, a diferencia de los estudiantes que no tienen mayor conocimiento de la misma.

Finalmente, es muy importante terminar este análisis de la actitud de los docentes y estudiantes hacia la ética con la reflexión que el Dr. Ortiz ${ }^{(13,14)}$ 
realizó en su artículo "Necesidad de un enfoque ético de la educación en medicina" donde remarca que "ciertas fallas del proceso educativo, que no son ciertamente los procedimientos de enseñanza-aprendizaje, sino una inadecuada conceptuación acerca de la naturaleza del hombre, en particular sobre qué es realmente la personalidad en formación. Tomando en cuenta esta situación, se fundamenta y sugiere la necesidad de aplicar una teoría ética y un modelo del desarrollo moral de la personalidad orientada a la moralización de la sociedad, como base de una educación del estudiante de medicina más consecuente con las necesidades de salud de nuestros países en desarrollo".

\section{REFERENCIAS BIBLIOGRÁFICAS}

1. Universidad de Navarra. Código Mundial Ética. Disponible en http://www.unav.es/cdb/ammlondres1.html.

2. Encolombia. Temas de Ética Médica. Disponible en http://www.encolombia.com/libreria-digital/ medicina/etica-medica/etica-medica
3. Perales A, Mendoza A. El mercado como determinante de inconducta médica. 2009. Doctorado en Medicina. Curso de Ética Médica. Facultad de Medicina. UNMSD. Lima. Perú.

4. Perales A. Salud mental: variables socioeconómicas, politicas y culturales. Anales de Salud Mental. 1993;10(1 y 2):83-107

5. Perales A, Mendoza A, Ortiz P. El mercado profesional como determinante de inconducta médica. An Fac med. 2000;61(3):207-18.

6. Ellis SJ. The corrupting of the medical profession. Br J Clin Pract. 1999 Jan-Feb;51(1):4.

7. Forsman B. An ethical analysis of the phenomenon of misconduct in research Acta Oncol. 1999;38(1):107-10.

8. Gonzales A. Dimensiones de la Corrupción. Rev Psicol Univ Católica. 1985;3(1):39-50.

9. López E. Ética médica. 1983. Disponible en http:// www.uaca.ac.cr/bv/ebooks/salud/1.pdf

10. Universidad de Chile. Principios de ética médica. Disponible en http://www.uchile.cl/portal/investigacion/76033/principios-de-etica-medica.

11. Maglio F. Ética médica frente al paciente crítico. Disponible en http://www.intramed.net/contenidover.asp?contenidolD=81867.

12. Crivos M. Estudio antropológico de una sala de hospital. Med Soc. 1988;11:127-37.

13. Ortiz P. Cerebro y moral. 2000: 437-43. Anales del XVIII Congreso Peruano de Psiquiatría y III Reunión Regional Bolivariana de la APAL. Disponible en http://www.tarpuq.com/sites/default/files/ blogPOC/neurociencias/Etica\%20CEREBRO\%20 Y\%20MORAL.pdf.
14. Ortiz P. Aspectos éticos de la decisión terapéutica. Rev Salud Medicam. 1997;(37):28-31. Disponible en http://www.cuerpomedicorebagliati.org/agosto2009/material_seminario/textos/10.pdf.

15. Cortina, A. Ética mínima. Introducción a la filosofía práctica. Madrid. España: Ed. Tecnos. 2000.

16. Perales A. Problemas de comunicación en el equipo psiquiátrico. Rev Sanidad FFPP. 1986.

17. Ortiz P. Cuadernos de Psicobiología Social 6. E nivel consciente de la actividad personal. Perú, 2004:30

18. Ortiz P. Cuadernos de Psicobiología Social 1 , Introducción a una psicobiologia del hombre. Perú, 2004:164-5.

Artículo recibido el 20 de abril de 2015 y aceptado para publicación el 27 de mayo de 2015.

Conflicto de interés:

Los autores declaran que no existen conflictos de interés.

Financiamiento propio.

Correspondencia:

Dr. Manuel H. Izaguirre Sotomayor

Cel: 998445293 RPM: \#791660

Correo electrónico: manuel.izaguirre@gmail.com 